\title{
Developing a program to detect plastic bags hanging on overhead railway power lines through the use of drone photography
}

\author{
Naoki Matsuura* and Jue Zhang ** \\ * Kogakuin University, 1-24-2 Nishishinjuku, Shinjuku-ku, Tokyo 163-8677, Japan \\ em19020@ns.kogakuin.ac.jp \\ ** Kogakuin University, 1-24-2 Nishishinjuku, Shinjuku-ku, Tokyo 163-8677, Japan \\ zhangjue@cc.kogakuin.ac.jp
}

\begin{abstract}
There is an ongoing problem affecting railway companies, caused by plastic garbage flying on to overhead power lines. When such an issue occurs, the general way to deal with it is as follows - when a station employee receives a notice, they will patrol the area by foot to visually confirm the issue. However, there are several problems with this method, as secondary hazards may subsequently occur, and a significant amount of time may occur before the plastic hazard is discovered. Meanwhile, the railway companies, in an effort to deal with this issue, have begun practical field tests using drone photography. However, the footage shot by the drones will still need to be verified by human eyes, and currently, there are no methods of automated detection of plastic hazards being conducted at this time. This report will explore the development of a method enabling the automated detection of such plastic hazards on overhead wires through binary coded processing, using aerial footage shot by drones, and investigate the benefits of such a method. Furthermore, we considered the practical applications of such a method, and developed a GUI program that will be compatible with several different formats of image sizes.
\end{abstract}

Keywords: Image recognition, Drone, Program development

\section{INTRODUCTION}

For quite some time, railway companies have been dealing with the problem of plastic garbage flying onto overhead power lines, which can lead to hazards such as electrocution, and have been calling out to alert the public of this danger [1]. When such an issue occurs, the general way to deal with it is as follows - when a station employee receives a notice, they will patrol the area by foot to visually confirm the location of the plastic hazard. However, if the location of the problem cannot be clearly confirmed, not only will it take a significant amount of time to patrol the area by foot and make a visual confirmation, there is the possibility of a secondary hazard, perhaps due to the shape of the plastic and the surrounding environment. In order to resolve such issues, in recent years the railway companies have begun field tests using drones - normally used to determine the situation during railway accidents and disasters - to film footage of these incidents [2]. These tests are being conducted by assuming the various environments and circumstances that can occur with train car sheds, by flying the drones through various different types of flights and movements, with the intent of realizing an efficient and safe method of investigation. However, the test footage filmed by these drones are being verified by human eyes, and so no automated detection methods of abnormalities in the power lines are being conducted at this time. Therefore, our objective for this research is to use footage of the overhead power lines filmed by the drones to develop an automated method of detection of plastic bags and other hazards, and to create a GUI detection program for practical field use.

\section{METHODS OF DETECTION OF PLASTIC HAZARDS THAT HAVE ATTACHED TO OVERHEAD POWER LINES}

\subsection{Pixel value comparison of photos featuring plastic bags and photos of the backgrounds of overhead power lines}

In order to begin the process of the detection of plastic bags, we photographed plastic bags under 3 different outdoor light conditions - "clear daytime conditions", "cloudy daytime conditions", and "clear conditions immediately before sundown". We then converted the 
photos to grayscale, and measured the maximum and minimum pixel values. The plastic bags measured 57.0 $\mathrm{cm}$ in height, and $33.5 \mathrm{~cm}$ in width, were semi-transparent and white in color, without any text or symbols printed upon their surfaces (Figure 1). In order to measure the luminance of each outdoor light condition, we used the LM-8102 multi-environmental light meter for each condition.

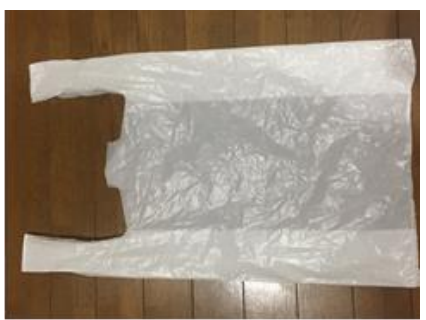

Figure 1: The plastic bag used in this experiment

10 photos were shot under each light condition, for a total of 30 photos. The photos were then measured for their maximum and minimum pixel values, and the results were displayed on Table 1. The luminance levels under "clear daytime conditions" measured over 20,000 lux, "cloudy daytime conditions" measured 643 lux, while "clear conditions immediately before sundown" measured 155 lux. As can be seen from Table 1, upon deriving an upper probability rate of $5 \%$ for the minimum value under each condition, that number was less than 130 lux for each condition.

Table 1: Average values for the maximum and minimum pixel values under each outdoor light condition

\begin{tabular}{|c|c|c|c|}
\hline & $\begin{array}{c}\text { clear } \\
\text { daytime }\end{array}$ & $\begin{array}{c}\text { cloudy } \\
\text { daytime }\end{array}$ & $\begin{array}{c}\text { clear } \\
\text { conditions } \\
\text { immediately } \\
\text { before } \\
\text { sundown }\end{array}$ \\
\hline maximum & 255.00 & 234.60 & 238.40 \\
\hline minimum & 71.75 & 95.22 & 87.50 \\
\hline
\end{tabular}

In light of these results, in order to enable the detection of plastic bags on overhead power lines from photos taken from the sky using binary coded processing to isolate the plastic bags from the background, we brought out photos of the background areas under overhead power lines under each outdoor light condition, together with the previously mentioned photos of the plastic bags, for a total of 60 photos. We applied binary image processing at threshold levels on 3 levels - 130, 150, and 170, and examined the proportion of pixels that were determined to be of a white color. Because under Japanese laws there are strict regulations that would not easily allow aerial drone photography of the overhead power lines, in order to shoot the background photos, we filmed from atop a bridge between Tobu-Nerima and Kamitabashi stations on the Tobu Tojo Line. From Figure 2, we can see that the proportion of pixels determined to be the color white in the images featuring the plastic bag is larger in the decreased level range of 150 to 170 , than the threshold level between 130 and 150 under all conditions. Furthermore, we can also see that the proportion of pixels determined to be the color white in the photos of the backgrounds is smaller in the decreased level range of 150 to 170 than the threshold level of 130 to 150 under all conditions, excepting clear daytime conditions. For clear daytime conditions, when comparing the photos of the plastic bag to the photos of the backgrounds in the decreased level range of the threshold level of 150 to 170, we found that the decreased levels of the plastic bag were larger. From these results, when considering the distribution of pixels under the proportional divergences of decreased levels were over or under a threshold level of 150 , we can surmise that the proportion of white pixels coming from the plastic bag to be a high value, while the proportion of white in the background photos to be lower, making the threshold level of 150 to be a desirable point of reference as a determining factor.

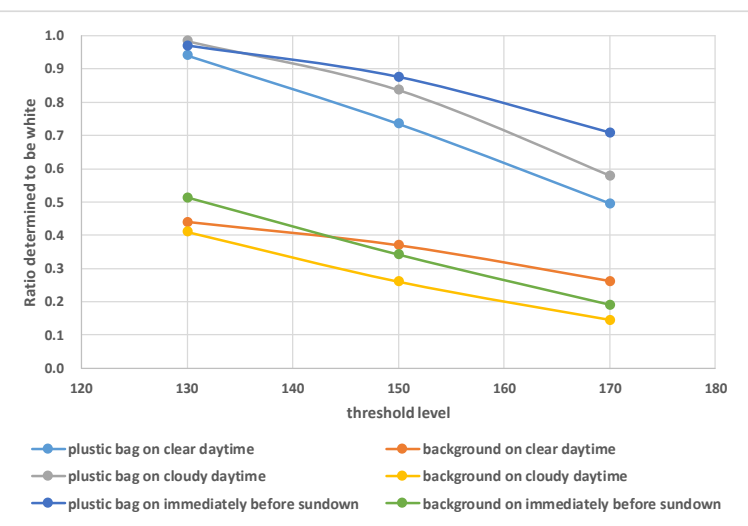

Figure 2: The proportional shift of determinations of white pixels under all conditions

\subsection{Program algorithm}

For this program, we first conducted binary image processing on the imported images. In light of the results derived from comparing the photos of the plastic bag and the background photos of the overhead power lines, we decided to use a threshold level of 150 . We then conducted a search consisting of blocks of a certain segment width along the horizontal band of each binary image derived from that process, and examined the proportion of pixels that resulted from the search and were determined to be the color white. If that proportion exceeded the judgment value, we would then branch off into a search along the vertical band of the images. If it did 
not exceed the judgment value, we would move forward $10 \mathrm{px}$ and continue the same process. Because the judgment value from this branched search had, determined from the results of Figure 2, exceeded a proportion of 0.7 for all of the images of the plastic bag under all outdoor light conditions, we set it as 0.7. After branching in the vertical direction, we transitioned one equivalent segment width lower at a time, conducting comparisons to the judgment value of 0.7 , and similarly to the comparison of when the vertical segment width and the horizontal segment width became equal, we decided that when the proportion of pixels determined to be white exceeded the judgment value of 0.7 , that a plastic bag had been detected. Furthermore, in order to minimize the processing time, and in order to avoid a duplication of areas where a detection had already been made, after a detection had been confirmed, the pixel value of that area of the image would be converted to 0 . The areas within the image that indicated a detection would be displayed in blue, so that a visual confirmation could be made after processing the search.

With regard to the segment width of the program, we decided that when conducting a search on photos taken at 3 different stages of vertical height by a drone, we would require an appropriate value for each condition.

\section{VERIFICATION TEST OF THE PLASTIC BAG DETECTION PROGRAM}

\subsection{Test method}

Because it would have been difficult to attach a plastic bag to the overhead power lines and photograph it with a drone, we went to the 1 st floor atrium of Kogakuin University, where we laid out a photo of the background (taken in advance for this test, and printed out to scale) on the floor, and stretched out black wires at a height of 5 meters (the same height as the power lines), with a plastic bag attached to the wires. Thus, we had recreated an outdoor condition for the purposes of this test. (Figure 3)

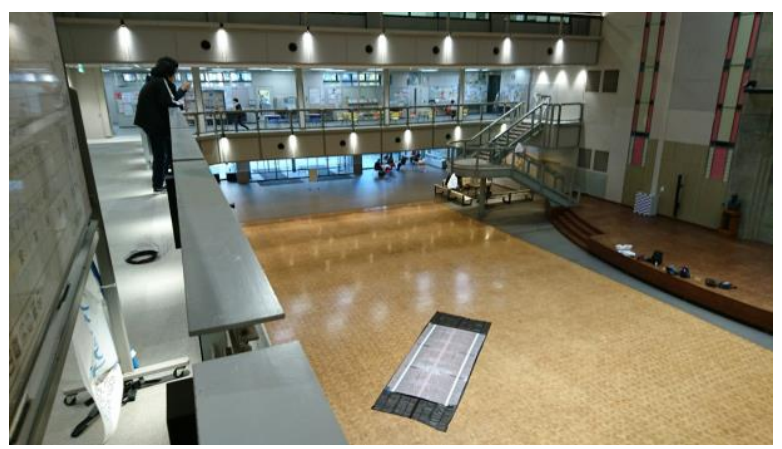

Figure 3: Photo of the drone photography test
We photographed this image using the Bebop 2 drone made by Parrot. The paper used for the background image turned out to have a high rate of reflectance, and generally reflected a high amount of white light. After taking the photos, we processed the image in order to better approximate conditions in the real world, by isolating the plastic bag and re-compositing the background image at scale. We took $10 \mathrm{HD}$ resolution photos at 3 different height ranges (measured from the floor) $-5.5 \mathrm{~m}-6.0 \mathrm{~m}$, $6.5 \mathrm{~m}-7.5 \mathrm{~m}, 9.5 \mathrm{~m}-10.0 \mathrm{~m}-\mathrm{a}$ total of 30 photos. For the detection tests, we utilized 6 different segment widths of 20px, 30px, 40px, 50px, 60px, and 70px. It was difficult to recreate the outdoor light conditions while indoors. On the day of the test, we used the LM-8102 multi-environmental light meter to measure the luminance of the atrium area, which resulted in a measurement of 664 lux, a reading which most closely approximated cloudy conditions. We therefore decided to conduct the test simulating only cloudy daytime conditions. The plastic bag photographed in this test measured $45 \mathrm{~cm}$ in height and $21 \mathrm{~cm}$ in width, and displayed the same other characteristics as the bag used in Figure 1.

\subsection{Test result and observations}

Table 2 displays the detection rate at each height range and segment width. Figure 4 depicts an example of a photo image shot from a height range of $6.5 \mathrm{~m}-7.5 \mathrm{~m}$, and analyzed by the program at a segment width of 40px. As can be seen from Table 2, at segment widths of 30px and $40 \mathrm{px}$, this test shows that it is possible to derive a highly precise rate of detection of plastic bags at all height ranges.

Table 2: Detection rate of plastic bag under each condition

\begin{tabular}{|c|c|c|c|c|c|c|}
\hline height & $20 \mathrm{px}$ & $30 \mathrm{px}$ & $40 \mathrm{px}$ & $50 \mathrm{px}$ & $60 \mathrm{px}$ & $70 \mathrm{px}$ \\
\hline $5.5-6.5 \mathrm{~m}$ & 0 & 100 & 100 & 100 & 100 & 100 \\
\hline $6.5-7.5 \mathrm{~m}$ & 40 & 100 & 100 & 100 & 100 & 100 \\
\hline $7.5-10.0 \mathrm{~m}$ & 100 & 100 & 100 & 40 & 0 & 0 \\
\hline
\end{tabular}

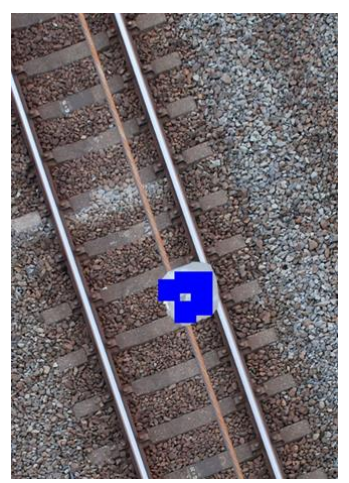

Figure 4: Example of detection of plastic bag 


\section{A GUI PROGRAM THAT CAN ISOLATE AND DETECT PLASTIC BAGS FROM THE BACKGROUND USING PHOTOS OF THE OVERHEAD POWER LINES}

\subsection{Program overview}

From the standpoint of practicality, we created a GUI program that could automatically optimize segment widths to adjust for varying pixel counts, in order to be compatible with images photographed by drones that differed from the type we used for this test. Figure 5 depicts a screenshot during the execution of this program. The user selects the type of device (drone) used by choosing one of the buttons arranged at the top left portion of the screen. After making a selection, the user will then enter the file number and number of files he or she wishes to process into the text box located at the bottom of the screen. Finally, the user will click the Run button. When the analysis is concluded, the results indicating whether a plastic bag has been detected will be displayed to the right of the Run button.

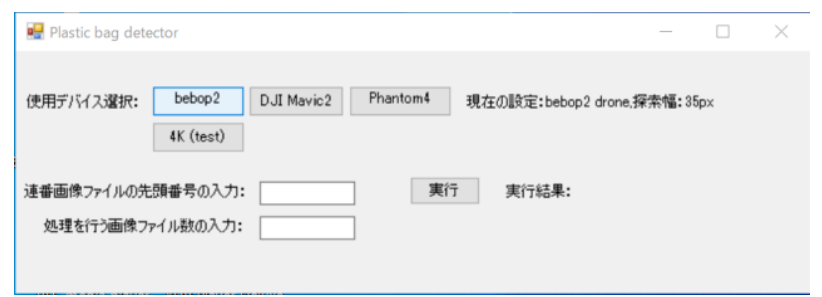

Figure 5: Screenshot following execution of the program

\subsection{Correcting determining segment widths for image} sizes

Because the resolution and field of view can vary depending on the type of camera equipped by a drone, we made a calculation which would correct for determining segment widths, as shown in Formula (1). The corrected determining segment width is $\mathrm{x}$, the horizontal pixel number of the image is $n$, and the field of view of the drone's camera is $\theta$.

$$
x=\frac{n}{1920} \times \frac{\tan 0.698}{\tan \left(\frac{\theta}{2} \times \frac{\pi}{180}\right)} \times 35
$$

Using each of the parameters of the Bebop 2 drone as a standard, and deriving comparisons of pixel numbers from other devices (drones) and fields of view from other cameras, we applied a correction to the standard determining segment width of 35 (the plastic bag detection program's standard as set by the Bebop 2 drone).

Because we were not able to access any other drones except the Bebop 2, as a means of verifying if this correction of the determining segment width was being properly reflected, we presumed that all fields of view were identical, enlarged the trimmed images used in this test to a proportional size of 5472 x 3078, with any marginal or blank spaces assigned a pixel value of 0 and thus, we were able to conduct this test having processed the images at a simulated $4 \mathrm{~K}$ size. As a result, because there were no significant differences in the detection results between the $4 \mathrm{~K}$ images and full HD images, we were able to assume that the corrected determining segment widths were valid (Figure 6).

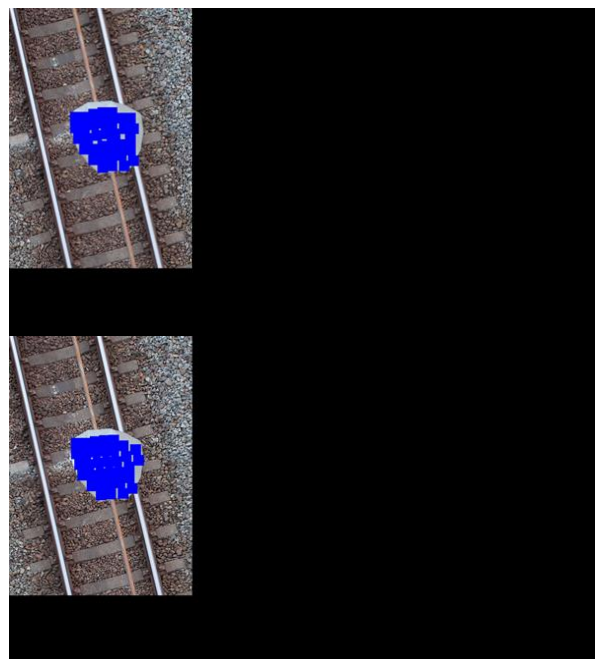

Figure 6: Examples of analysis results of a full HD image (above), and a 4K image (below)

\section{CONCLUSIONS}

In this paper, we focused on luminosity, and created a GUI program that was compatible with varying types of image sizes, and examined the ways we could detect plastic hazards from aerial photos taken of overhead power lines. As a result, we were able to determine that we could detect plastic hazards at a highly precise rate, using binary image processing, with a detection program set to appropriate segment widths, within a height range of 5.5 meters to 10.0 meters under cloudy daytime conditions.

\section{REFFERENCES}

[1]

http://www.jrkyushu.co.jp/company/csr/safety/attenti on/shock.html

[2]

https://cweb.canon.jp/newsrelease/2017-11/pr-droneexperiment.html 